\title{
An Explicit Example of Polynomials Orthogonal on the Unit Circle with a Dense Point Spectrum Generated by a Geometric Distribution
}

Alexei ZHEDANOV

School of Mathematics, Renmin University of China, Beijing 100872, China

E-mail: zhedanov@ruc.edu.cn

Received November 02, 2020, in final form December 19, 2020; Published online December 21, 2020 https://doi.org/10.3842/SIGMA.2020.140

\begin{abstract}
We present a new explicit family of polynomials orthogonal on the unit circle with a dense point spectrum. This family is expressed in terms of $q$-hypergeometric function of type ${ }_{2} \phi_{1}$. The orthogonality measure is the wrapped geometric distribution. Some "classical" properties of the above polynomials are presented.
\end{abstract}

Key words: polynomials orthogonal on the unit circle; wrapped geometric dustribution; dense point spectrum

2020 Mathematics Subject Classification: 33D45; 42C05

\section{Introduction}

Let $\Phi_{n}(z)$ be monic polynomials $\Phi_{n}(z)=z^{n}+O\left(z^{n-1}\right)$ defined through the recurrence relation $[17]$

$$
\Phi_{n+1}(z)=z \Phi_{n}(z)-\bar{a}_{n} \Phi_{n}^{*}(z), \quad \Phi_{0}(z)=1,
$$

where

$$
\Phi_{n}^{*}(z)=z^{n} \bar{\Phi}_{n}(1 / z)
$$

and where $\bar{\Phi}_{n}(z)$ means complex conjugation of expansion coefficients of the polynomial $\Phi_{n}(z)$. The recursion parameters

$$
a_{n}=-\bar{\Phi}_{n+1}(0)
$$

are called the Verblunsky (sometimes also reflection, Schur etc.) parameters [17].

Under the condition

$$
\left|a_{n}\right|<1, \quad n=0,1,2, \ldots
$$

the polynomials $\Phi_{n}(z)$ are orthogonal on the unit circle with respect to a positive measure $\mathrm{d} \sigma(\theta)$

$$
\int_{0}^{2 \pi} \Phi_{n}\left(\mathrm{e}^{\mathrm{i} \theta}\right) \bar{\Phi}_{m}\left(\mathrm{e}^{-\mathrm{i} \theta}\right) \mathrm{d} \sigma(\theta)=h_{n} \delta_{n m}
$$

where

$$
h_{n}=\left(1-\left|a_{0}\right|^{2}\right)\left(1-\left|a_{1}\right|^{2}\right) \cdots\left(1-\left|a_{n-1}\right|^{2}\right)
$$

are normalization constants (which are nonzero due to condition (1.1)). In this case $\Phi_{n}(z)$ are called the orthogonal polynomials on the unit circle (OPUC). 
Note that orthogonality relation (1.2) is equivalent to conditions [17]

$$
I_{n j} \equiv \int_{0}^{2 \pi} \Phi_{n}\left(\mathrm{e}^{\mathrm{i} \theta}\right) \mathrm{e}^{-\mathrm{i} j \theta} \mathrm{d} \sigma(\theta)=h_{n} \delta_{n j}, \quad j=0,1,2, \ldots, n .
$$

Equivalently, OPUC $\Phi_{n}(z)$ can be constructed in terms of trigonometric moments $\sigma_{n}$. The latter are defined as

$$
\sigma_{n}=\int_{0}^{2 \pi} \mathrm{e}^{\mathrm{i} n \theta} \mathrm{d} \sigma(\theta), \quad n=0, \pm 1, \pm 2, \ldots
$$

Then polynomials $\Phi_{n}(z)$ have the explicit expression

$$
\Phi_{n}(z)=\left(\Delta_{n}\right)^{-1}\left|\begin{array}{cccc}
\sigma_{0} & \sigma_{1} & \ldots & \sigma_{n} \\
\sigma_{-1} & \sigma_{0} & \ldots & \sigma_{n-1} \\
\ldots & \ldots & \ldots & \ldots \\
\sigma_{1-n} & \sigma_{2-n} & \ldots & \sigma_{1} \\
1 & z & \ldots & z^{n}
\end{array}\right|
$$

where

$$
\Delta_{n}=\left|\begin{array}{cccc}
\sigma_{0} & \sigma_{1} & \ldots & \sigma_{n-1} \\
\sigma_{-1} & \sigma_{0} & \ldots & \sigma_{n-2} \\
\ldots & \ldots & \ldots & \ldots \\
\sigma_{1-n} & \sigma_{2-n} & \ldots & \sigma_{0}
\end{array}\right|
$$

are Toeplitz determinants which are all positive $\Delta_{n}>0, n=0,1,2, \ldots$ Note the symmetry property of the trigonometric moments

$$
\sigma_{-n}=\bar{\sigma}_{n}
$$

Explicit examples of polynomials orthogonal on unit circle are very interesting from different point view. By "explicit examples" we mean that all main objects: the parameters $a_{n}$, the moments $\sigma_{n}$, the measure $\sigma(\theta)$ and the polynomials themselves $\Phi_{n}(z)$ have explicit expressions in terms of special functions. Usually, in most known explicit examples the parameters $a_{n}$ are given by elementary functions of $n$ while the OPUC $\Phi_{n}(z)$ are expressed in terms of hypergeometric functions (either ordinary or basic). A list of known explicit examples can be found, e.g., in Simon's monograph [17].

In $[21,27]$ new explicit examples of OPUC were presented. In these examples polynomials $\Phi_{n}(z)$ are expressed in terms of elliptic hypergeometric function ${ }_{3} E_{2}(z)$ while the moments $\sigma_{n}$ and the recurrence parameters $a_{n}$ have simple expressions in terms of elliptic functions. The most interesting property of the OPUC of these examples is that they are orthogonal on the unit circle with respect to a dense point measure. This means that the function $\sigma(\theta)$ is a step function with infinitely many points $\theta_{s}$ of jumps, and these points are dense on the interval $[0,2 \pi]$. In terms of the distribution function this can be presented as

$$
\rho(\theta)=\sum_{s=-\infty}^{\infty} M_{s} \delta\left(\theta-\theta_{s}\right),
$$

where $\rho(\theta)$ is a distribution defined as $\mathrm{d} \sigma(\theta)=\rho(\theta) \mathrm{d} \theta, \delta(\theta)$ is the Dirac delta function and $M_{s}$ are concentrated masses located at points of jumps $\theta_{s}$. The spectral points $z_{s}=\exp \left(\mathrm{i} \theta_{s}\right)$ are dense on the unit circle. 
Then orthogonality relation (1.2) can then be presented as

$$
\sum_{s=-\infty}^{\infty} M_{s} \Phi_{n}\left(\mathrm{e}^{\mathrm{i} \theta_{s}}\right) \bar{\Phi}_{m}\left(\mathrm{e}^{-\mathrm{i} \theta_{s}}\right)=h_{n} \delta_{n m}
$$

From general considerations (see, e.g., [17]) it follows that polynomials orthogonal with respect to such dense point measures are rather generic if one assumes some natural restrictions upon behavior of the recurrence parameters $a_{n}$. On the other hand, such measures are very important from physical point of view, because they correspond to the phenomenon of the Anderson localization $[10,17]$.

Usually examples of OPUC with dense point spectrum are related to sequences of the parameters $a_{n}$ which behave (quasi) stochastically inside the interval $\left|a_{n}\right|<1$ [17]. OPUC in [21, 27] provide perhaps the first known examples of a pure point dense measure on the unit circle where both the coefficients $a_{n}$ and the moments $\sigma_{n}$ are given explicitly by analytic functions in $n$.

In this paper we propose a much simpler explicit example of polynomials orthogonal on the unit with respect to a (wrapped) geometric distribution which is dense on the unit circle. Polynomials themselves are expressed in terms of basic hypergeometric function ${ }_{2} \phi_{1}(q ; z)$ with $|q|=1$.

\section{Wrapped geometric distribution and corresponding OPUC}

Let $q$ be a fixed point belonging to the unit circle $|q|=1$ and not a root of unity, i.e., we demand that $q^{n} \neq 1$ for all natural integer $n=0,1, \ldots$. Choose a real parameter $p$ within the unit interval $0<p<1$. Define the measure on the unit circle as

$$
\rho(\theta)=(1-p) \sum_{s=0}^{\infty} p^{s} \delta(\theta-s \chi)
$$

where $\chi$ is a fixed irrational parameter $0<\chi<1$ such that

$$
q=\exp (2 \pi \mathrm{i} \chi) .
$$

Irrationality of $\chi$ means that the set of points $z_{s}=q^{s}, s=0,1, \ldots$ (i.e., the location of jumps of the measure) is dense on the unit circle. The weights (i.e., the concentrated masses) $w_{s}$ at the points $z_{s}$ form the geometric sequence: $w_{s}=p^{s}, s=0,1,2, \ldots$

Corresponding trigonometric moments are

$$
\sigma_{n}=\sum_{s=0}^{\infty} z_{s}^{n} w_{s}=(1-p) \sum_{s=0}^{\infty} q^{s n} p^{s}=\frac{1-p}{1-p q^{n}}, \quad n=0, \pm 1, \pm 2, \ldots
$$

Note that the measure (2.1) can be interpreted as the wrapped geometric distribution on the unit circle (see, e.g., $[8,15]$ for definition and discussion of wrapped distributions on the unit circle).

Relation $\sigma_{0}=1$ means that the measure (2.1) has the standard normalization condition.

One can present expression (2.3) as

$$
\sigma_{n}=\frac{(p ; q)_{n}}{(p q ; q)_{n}}
$$

where the $q$-shifted factorial $\left(q\right.$-Pochhammer symbol) is defined as $[4,9](a ; q)_{0}=1$ and

$$
(a ; q)_{n}=(1-a)(1-a q) \cdots\left(1-a q^{n-1}\right)
$$


for positive $n=1,2, \ldots$ and

$$
(a ; q)_{n}=\frac{1}{\left(a q^{n} ; q\right)_{-n}}
$$

for negative $n=-1,-2, \ldots$

It is known that the Laurent biorthogonal Pastro polynomials $P(z ; a, b)$ [16] depending on two arbitrary parameters $a, b$ can be uniquely defined through their moments [22]

$$
\sigma_{n}=\frac{(a ; q)_{n}}{(b ; q)_{n}}, \quad n=0, \pm 1, \pm 2, \ldots
$$

Explicitly, these polynomials are given by [16, 22]

$$
P(z ; a, b)=\mu_{n 2} \phi_{1}\left(\begin{array}{l}
q^{-n}, b \\
a q^{1-n}
\end{array} ; z\right)
$$

where $\mu_{n}$ is an appropriate normalization factor to fulfill the condition $P_{n}(z)=z^{n}+O\left(z^{n-1}\right)$. The definition and notation of the basic hypergeometric function ${ }_{m} \phi_{n}(z)$ is standard (see, e.g., $[4,9])$. For example, in the special case $m=n+1$ we have the expressions

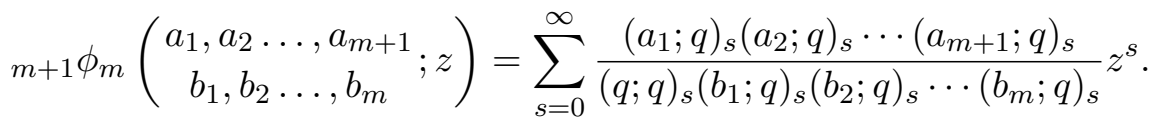

Note the Laurent biorthogonal polynomials (LBP) can be considered as a generalization of the OPUC. Their main distinction from OPUC is that the moments $\sigma_{n}$ do not satisfy, in general, the symmetry condition (1.5). The LBP can also be characterized by the three-term recurrence relation of $R_{I}$ type $[7,24]$

$$
P_{n+1}(z)+g_{n} P_{n}(z)=z\left(P_{n}(z)+d_{n} P_{n-1}(z)\right), \quad P_{0}=1, \quad P_{-1}=0
$$

with some recurrence coefficients $g_{n}, d_{n}$.

In contrast to the case of orthogonal polynomials, the recurrence relation (2.7) can be presented in the form of the generalized eigenvalue problem [25]

$$
J_{1} \mathbf{P}(z)=z J_{2} \mathbf{P}(z)
$$

where $J_{1}, J_{2}$ are upper and lower bidiagonal matrices acting on the vector

$$
\mathbf{P}(z)=\left(P_{0}(z), P_{1}(z), \ldots\right) .
$$

Comparing expressions (2.5) and (2.4) we can conclude that the OPUC corresponding to the wrappedd geometric distribution are special case of the Pastro polynomials with $a=p, b=q p$.

This allows us to present the main result

Theorem 2.1. The polynomials $\Phi_{n}(z)$ orthogonal on the unit circle with respect to the wrapped geometric distribution (2.1) have the explicit expression

$$
\Phi_{n}(z)=\mu_{n 2} \phi_{1}\left(\begin{array}{l}
q^{-n}, p q \\
p q^{1-n}
\end{array} ; z q\right)
$$

where

$$
\mu_{n}=q^{-n} \frac{(q ; q)_{n}\left(p q^{1-n} ; q\right)_{n}}{\left(q^{-n} ; q\right)_{n}(p q ; q)_{n}}=p^{n} \frac{\left(p^{-1} ; q\right)_{n}}{(q p ; q)_{n}} .
$$


One can directly check that the polynomials (2.9) satisfy orthogonality relations (1.4). Indeed, one has

$$
I_{n j}=(1-p) \sum_{s=0}^{\infty} \Phi_{n}\left(q^{s}\right) q^{-s j} p^{s}=(1-p) \mu_{n} \sum_{s=0}^{\infty} \sum_{k=0}^{n} \frac{\left(q^{-n} ; q\right)_{k}(p q ; q)_{k}}{(q ; q)_{k}\left(p q^{1-n} ; q\right)_{k}} q^{(1+s) k} q^{-j s} p^{s} .
$$

Performing summation over $s$ we get

$$
I_{n j}=\frac{(1-p) \mu_{n}}{1-p q^{-j}} \sum_{k=0}^{n} \frac{\left(q^{-n} ; q\right)_{k}(p q ; q)_{k}\left(p q^{-j} ; q\right)_{k}}{(q ; q)_{k}\left(p q^{1-n}\right)_{k}\left(p q^{1-j} ; q\right)_{k}} q^{k}=\frac{(1-p) \mu_{n}}{1-p q^{-j}}{ }_{3} \phi_{2}\left(\begin{array}{c}
q^{-n}, p q, p q^{-j} \\
p q^{1-n}, p q^{1-j}
\end{array} ; q\right) .
$$

The above expression can be simplified by the $q$-Saalschütz formula [4, 9]:

$$
{ }_{3} \phi_{2}\left(\begin{array}{c}
q^{-n}, a, b \\
c, a b c^{-1} q^{1-n}
\end{array} ; q\right)=\frac{(c / a ; q)_{n}(c / b ; q)_{n}}{(c ; q)_{n}(c /(a b) ; q)_{n}} .
$$

We thus have

$$
I_{n j}=\frac{(1-p) \mu_{n}}{1-p q^{-j}} \frac{\left(q^{-n} ; q\right)_{n}\left(q^{j+1-n} ; q\right)_{n}}{\left(p q^{1-n} ; q\right)_{n}\left(p^{-1} q^{j-n} ; q\right)_{n}} .
$$

The factor $\left(q^{j+1-n} ; q\right)_{n}$ in $(2.11)$ becomes zero when $j=0,1, \ldots, n-1$ and hence

$$
I_{n j}=0, \quad j=0,1, \ldots, n-1,
$$

which is equivalent to orthogonality relation (1.4). It remains to show that $I_{n n}>0$. After simple calculations one can arrive at the expression

$$
I_{n n}=h_{n}=\frac{\left|(q ; q)_{n}\right|^{2}}{\left|(p q ; q)_{n}\right|^{2}} p^{n}
$$

from which it is clear that $h_{n}>0$ for all $n=0,1,2, \ldots$ due to condition $q^{n} \neq 1$.

Explicit expression for the the recurrence parameters $a_{n}$ follows from (2.9) and (2.10):

$$
\bar{a}_{n-1}=-\Phi_{n}(0)=-\mu_{n}=-p^{n} \frac{\left(p^{-1} ; q\right)_{n}}{(q p ; q)_{n}} .
$$

For the square of absolute values we have rather simple expression

$$
\left|a_{n-1}\right|^{2}=\bar{a}_{n-1} a_{n-1}=\frac{(1-p)^{2}}{1+p^{2}-p\left(q^{n}+q^{-n}\right)}=\frac{1}{1+\beta \sin ^{2}(\chi \pi n)},
$$

where

$$
\beta=\frac{4 p}{(1-p)^{2}}
$$

and where the parameter $\chi$ is the same as in (2.2).

It is seen from (2.13) that the values $\left|a_{n}\right|$ oscillate inside the interval

$$
\frac{1-p}{1+p}<\left|a_{n}\right|<1, \quad n=0,1, \ldots
$$

Because of irrationality of $\chi$ the absolute value $\left|a_{n}\right|$ achieves the boundaries of this interval with any prescribed accuracy (never achieving exact boundary values). Note that $a_{-1}=-1$ which corresponds to the standard initial conditions for OPUC [5, 17]. It is easily verified that expression (2.12) for $h_{n}$ agrees with relation (1.3).

The OPUC (2.9) can be considered as $|q|=1$ analogs of the OPUC introduced by Askey in [1] (see also [3] for more general OPUC of Askey's type). 


\section{3 "Classicality" of the polynomials $\Phi_{n}(z)$}

The OPUC (2.9) possess "classical" properties which make them similar to classical orthogonal polynomials.

First of all, they satisfy the three-term recurrence relation (2.7) where the recurrence coefficients are

$$
g_{n}=\frac{q^{n}-p}{1-p q^{n+1}}, \quad d_{n}=-\frac{p\left(1-q^{n}\right)^{2}}{\left(1-p q^{n}\right)\left(1-p q^{n+1}\right)} .
$$

Moreover, the polynomial $\Phi_{n}(z)$ possess a remarkable duality property. Indeed, one can rewrite polynomials $\Phi_{n}(z)$ in a different form

$$
\Phi_{n}(z)=p^{n} \frac{(q ; q)_{n}}{(p q ; q)_{n}} z_{3}^{n} \phi_{2}\left(\begin{array}{c}
q^{-n}, p^{-1}, z^{-1} \\
q, 0
\end{array} ; q\right),
$$

which can be obtained from (2.9) by standard transformation formulas [4, 9].

From this formula the duality property

$$
A_{s} \Phi_{s}\left(q^{n}\right)=A_{n} \Phi_{n}\left(q^{s}\right)
$$

follows, where

$$
A_{n}=\frac{(p q ; q)_{n}}{(q ; q)_{n}} p^{-n}
$$

This property resembles corresponding duality properties for the classical orthogonal polynomials from the Askey scheme $[2,11,19]$. The main difference is that the polynomials $\Phi_{n}(z)$ satisfy the generalized eigenvalue problem (2.8) instead of the ordinary eigenvalue problem for orthogonal polynomials.

From the duality property one can derive the second-order $q$-difference equation

$$
B_{s+1} \Phi_{n}\left(q^{s+1}\right)+g_{s} \Phi_{n}\left(q^{s}\right)=q^{n}\left(\Phi_{n}\left(q^{s}\right)+B_{s}^{-1} d_{s} \Phi_{n}\left(q^{s-1}\right)\right)
$$

where

$$
B_{s}=\frac{A_{s-1}}{A_{s}}=\frac{p\left(1-q^{s}\right)}{1-p q^{s}}
$$

Equation (3.3) can also be presented in the form of the generalized eigenvalue problem

$$
L \Phi_{n}(z)=q^{n} M \Phi_{n}(z)
$$

where the first-order $q$-difference operators $L, M$ act on the argument $z$ of the polynomials.

Relations (2.8) and (3.4) mean that the polynomials possess the bispectrality property: they satisfy simultaneously two GEVP. Concerning definition and general theory of bispectrality see, e.g., [6]. For orthogonal polynomials from the Askey scheme this property is well known [9]. For biorthogonal polynomials and rational functions the bispectrality is known for some special families. The most general from them are elliptic biorthogonal functions [18]. However the general theory of bispectrality for systems satisfying GEVP is not yet developed (see, e.g., [20, 23] for algebraic description of bispectrality on the "lowest" level of hypergeometric functions ${ }_{3} F_{2}(1)$ ).

The duality property implies that for $z=q^{s}, s=0,1,2, \ldots$ the hypergeometric function in (3.1) reduces to a polynomial of degree $s$ of the argument $q^{-n}$. 
It is well known (see, e.g., [17]) that if $z_{0}$ is a point on the unit circle corresponding to a concentrated mass $M_{0}$ then the relation

$$
\sum_{n=0}^{\infty} \frac{\left|\Phi_{n}\left(z_{0}\right)\right|^{2}}{h_{n}}=1 / M_{0}
$$

holds, where the normalization coefficient $h_{n}$ is defined in (1.3).

In our case this means that for every spectral point $z_{s}=q^{s}, s=0,1, \ldots$ there exists the identity

$$
\sum_{n=0}^{\infty} \frac{\left|\Phi_{n}\left(q^{s}\right)\right|^{2}}{h_{n}}=M_{s}^{-1}=\frac{p^{-s}}{1-p} .
$$

Identity (3.5) follows easily from the duality property (3.2) and from orthogonality relation.

So far, we have considered the case when $q$ is not a root of unity. If, otherwise, $q$ is a primitive root of unity

$$
q=\exp \left(\frac{2 \pi \mathrm{i} M}{N}\right)
$$

with coprime integers $M, N$, then there are only $N$ distinct mass points on the unit circle located at $z_{s}=q^{s}, s=0,1,2, \ldots, N-1$. In this case the polynomials $\Phi_{n}(z)$ are orthogonal on vertices of a regular $N$-gon with respect to the finite wrapped geometric distribution:

$$
\sum_{s=0}^{N-1} \Phi_{n}\left(q^{s}\right) \bar{\Phi}_{m}\left(q^{-s}\right)\left(1-p^{N}\right) p^{s}=h_{n} \delta_{n m}, \quad n, m=0,1, \ldots, N-1 .
$$

See [26] for other explicit examples of polynomials orthogonal on the vertices of regular polygons.

\section{Concluding remarks}

In contrast to examples of OPUC obtained in [27], the polynomials (2.6) have non-real moments $\sigma_{n}$ and hence the coefficients $a_{n}$ are non-real as well. This means that it is impossible to associate with OPUC (2.6) polynomials orthogonal on an interval of the real line. In [27] explicit examples of polynomials orthogonal with dense point spectrum on an interval were presented using standard Szegó mapping from OPUC to an interval of the real line. We mention also examples of OPUC and ordinary orthogonal polynomials with dense point spectrum presented in $[13,14]$.

The OPUC (2.6) allow a trivial modification which shifts all spectral points on the unit circle on the same constant angle $\varphi$, i.e., we can consider the same weights $w_{s}=p^{s}(1-p)$ located at the points

$$
\theta_{s}=2 \pi \chi s+\varphi, \quad s=0,1,2, \ldots
$$

Equivalently, this means that the new spectral points will be $\tilde{z}_{s}=\mathrm{e}^{\mathrm{i} \varphi} q^{s}, s=0,1,2, \ldots$

Such transformation is equivalent to a simple rotation of the argument of OPUC [5, 17]:

$$
\tilde{\Phi}_{n}(z)=\mathrm{e}^{-\mathrm{i} \varphi n} \Phi_{n}\left(\mathrm{e}^{\mathrm{i} \varphi} z\right) .
$$

Another modification of the OPUC (2.6) is more substantional. It leads to Laurent biorthogonal polynomials orthogonal on the unit circle with dense point measure. 
Indeed, assume that the spectral points on the unit circle are the same: $z_{s}=q^{s}, s=$ $0,1,2, \ldots$ Take the weights:

$$
w_{s}=p^{s} \frac{\left(q^{k} ; q\right)_{s}}{(q ; q)_{s}}, \quad 0<p<1, \quad k=1,2,3, \ldots
$$

For $k=1$ we return to the case of the wrapped geometric distribution. For $k>1$ the moments are

$$
\sigma_{n}=\sum_{s=0}^{\infty} \frac{\left(q^{k} ; q\right)_{s}}{(q ; q)_{s}} p^{s} q^{s n}
$$

By $q$-binomial theorem $[4,9]$ the above sum is simplified to

$$
\sigma_{n}=\frac{\left(q^{k} p q^{n} ; q\right)_{\infty}}{\left(p q^{n} ; q\right)_{\infty}}=\frac{1}{\left(p q^{n} ; q\right)_{k}}=\frac{(p ; q)_{n}}{(p ; q)_{k}\left(p q^{k} ; q\right)_{n}} .
$$

Remark. Usually, the convergence problem for $q$-series like (4.2) with $|q|=1$ is highly nontrivial (see, e.g., [12]). In our case however this problem does not appear because for integer $k$ there is cancellation of almost all terms (apart of a finite number of initial ones) in denominators of the coefficients in (4.2). Hence the convergence for $0<p<1$ still takes place.

For fixed $k$ the moments $\sigma_{n}$ coincide (up to a constant factor) with the moments (2.5) for the Pastro polynomials with $a=p, b=p q^{k}$. Hence from (2.6) we have explicit expression for them

$$
P(z)=\mu_{n 2} \phi_{1}\left(\begin{array}{c}
q^{-n}, p q^{k} \\
p q^{1-n}
\end{array} ; q z\right)
$$

where

$$
\mu_{n}=\frac{p^{n}\left(p^{-1} ; q\right)_{n}}{\left(p q^{k} ; q\right)_{n}}
$$

These polynomials are NOT OPUC (apart from the already considered case $k=1$ ) because the weights (4.1) are not positive and hence the moments $\sigma_{n}$ do not satisfy symmetry property (1.5).

Existence of other explicit examples of OPUC with dense point spectrum is an interesting open problem.

\section{Acknowledgements}

The author is indebted for F.A. Grünbaum, A. Magnus, V. Spiridonov, S. Tsujimoto and L. Vinet for discussions and for anonymous referees for valuable remarks. The author is gratefully holding Simons CRM Professorship and is funded by the National Foundation of China (Grant No. 11771015).

\section{References}

[1] Askey R., Comments to Gabor Szego” "Collected papers, Vol. 1", Contemporary Mathematicians, Birkhäuser, Boston, Mass., 1982, 806-811.

[2] Bannai E., Ito T., Algebraic combinatorics. I. Association schemes, The Benjamin/Cummings Publishing Co., Inc., Menlo Park, CA, 1984.

[3] Costa M.S., Godoy E., Lamblém R.L., Sri Ranga A., Basic hypergeometric functions and orthogonal Laurent polynomials, Proc. Amer. Math. Soc. 140 (2012), 2075-2089. 
[4] Gasper G., Rahman M., Basic hypergeometric series, 2nd ed., Encyclopedia of Mathematics and its Applications, Vol. 96, Cambridge University Press, Cambridge, 2004.

[5] Geronimus Ya.L., Polynomials orthogonal on a circle and their applications, Amer. Math. Soc. Translation 1954 (1954), 79 pages.

[6] Grünbaum F.A., The bispectral problem: an overview, in Special Functions 2000: Current Perspective and Future Directions (Tempe, AZ), NATO Sci. Ser. II Math. Phys. Chem., Vol. 30, Editors J. Bustoz, M.E.H. Ismail, S.K. Suslov, Kluwer Acad. Publ., Dordrecht, 2001, 129-140.

[7] Hendriksen E., van Rossum H., Orthogonal Laurent polynomials, Indag. Math. 89 (1986), 17-36.

[8] Jacob S., Jayakumar K., Wrapped geometric distribution: a new probability model for circular data, J. Stat. Theory Appl. 12 (2013), 348-355.

[9] Koekoek R., Lesky P.A., Swarttouw R.F., Hypergeometric orthogonal polynomials and their $q$-analogues, Springer Monographs in Mathematics, Springer-Verlag, Berlin, 2010, earlier edition available at https: //homepage.tudelft.nl/11r49/askey.html.

[10] Lagendijk A., van Tiggelen B., Wiersma D., Fifty years of Anderson localization, Phys. Today 62 (2009), no. $8,24-29$.

[11] Leonard D.A., Orthogonal polynomials, duality and association schemes, SIAM J. Math. Anal. 13 (1982), 656-663.

[12] Lubinsky D.S., Saff E.B., Convergence of Padé approximants of partial theta functions and the Rogers-Szegő polynomials, Constr. Approx. 3 (1987), 331-361.

[13] Magnus A.P., Special nonuniform lattice (snul) orthogonal polynomials on discrete dense sets of points, J. Comput. Appl. Math. 65 (1995), 253-265.

[14] Magnus A.P., Semi-classical orthogonal polynomials on the unit circle, Preprint MAPA 3072A, available at https://perso.uclouvain.be/alphonse.magnus/num3/m3xxx99.pdf.

[15] Mardia K.V., Jupp P.E., Directional statistics, Wiley Series in Probability and Statistics, John Wiley \& Sons, Ltd., Chichester, 2000.

[16] Pastro P.I., Orthogonal polynomials and some q-beta integrals of Ramanujan, J. Math. Anal. Appl. 112 (1985), 517-540.

[17] Simon B., Orthogonal polynomials on the unit circle. Part 1. Classical theory, American Mathematical Society Colloquium Publications, Vol. 54, Amer. Math. Soc., Providence, RI, 2005.

[18] Spiridonov V., Zhedanov A., Classical biorthogonal rational functions on elliptic grids, C. R. Math. Acad. Sci. Soc. R. Can. 22 (2000), 70-76.

[19] Terwilliger P., Two linear transformations each tridiagonal with respect to an eigenbasis of the other, Linear Algebra Appl. 330 (2001), 149-203, arXiv:math.RA/0406555.

[20] Tsujimoto S., Vinet L., Zhedanov A., An algebraic description of the bispectrality of the biorthogonal rational functions of Hahn type, Proc. Amer. Math. Soc., to appear, arXiv:2005.04217.

[21] Tsujimoto S., Zhedanov A., Elliptic hypergeometric Laurent biorthogonal polynomials with a dense point spectrum on the unit circle, SIGMA 5 (2009), 033, 30 pages, arXiv:0809.2574.

[22] Vinet L., Zhedanov A., Spectral transformations of the Laurent biorthogonal polynomials. II. Pastro polynomials, Canad. Math. Bull. 44 (2001), 337-345.

[23] Vinet L., Zhedanov A., A unified algebraic underpinning for the Hahn polynomials and rational functions, arXiv:2009.05905.

[24] Zhedanov A., The "classical" Laurent biorthogonal polynomials, J. Comput. Appl. Math. 98 (1998), 121147.

[25] Zhedanov A., Biorthogonal rational functions and the generalized eigenvalue problem, J. Approx. Theory 101 (1999), 303-329.

[26] Zhedanov A., On the polynomials orthogonal on regular polygons, J. Approx. Theory 97 (1999), 1-14.

[27] Zhedanov A., Elliptic polynomials orthogonal on the unit circle with a dense point spectrum, Ramanujan J. 19 (2009), 351-384, arXiv:0711.4696. 